\title{
Modeling Anomalous Diffusion by a Subordinated Integrated Brownian Motion
}

\author{
Long Shi ${ }^{1,2}$ and Aiguo Xiao ${ }^{1}$ \\ ${ }^{1}$ School of Mathematics and Computational Science, Xiangtan University, Xiangtan, Hunan 411105, China \\ ${ }^{2}$ School of Science, Central South University of Forest and Technology, Changsha, Hunan 410004, China \\ Correspondence should be addressed to Long Shi; slong8008@163.com
}

Received 6 February 2017; Accepted 23 March 2017; Published 4 April 2017

Academic Editor: Giorgio Kaniadakis

Copyright (c) 2017 Long Shi and Aiguo Xiao. This is an open access article distributed under the Creative Commons Attribution License, which permits unrestricted use, distribution, and reproduction in any medium, provided the original work is properly cited.

We consider a particular type of continuous time random walk where the jump lengths between subsequent waiting times are correlated. In a continuum limit, the process can be defined by an integrated Brownian motion subordinated by an inverse $\alpha$-stable subordinator. We compute the mean square displacement of the proposed process and show that the process exhibits subdiffusion when $0<\alpha<1 / 3$, normal diffusion when $\alpha=1 / 3$, and superdiffusion when $1 / 3<\alpha<1$. The time-averaged mean square displacement is also employed to show weak ergodicity breaking occurring in the proposed process. An extension to the fractional case is also considered.

\section{Introduction}

Anomalous diffusion is found in a wide diversity of systems (see review articles [1-4] and references therein). In one dimension, it is characterized by a mean square displacement (MSD) of the form

$$
\left\langle(\Delta x)^{2}\right\rangle(t) \propto K_{\alpha} t^{\alpha}
$$

with $\alpha \neq 1$, which deviates from the linear dependence on time found in normal diffusion. The coefficient $K_{\alpha}$ is generalized diffusion constant. It is called subdiffusion for $0<\alpha<1$ and superdiffusion for $\alpha>1$ [2].

A fundamental account to anomalous diffusion is provided by a stochastic process called continuous time random walk (CTRW), which was originally introduced by Montroll and Weiss in 1965 [5]. In a continuum limit, the process has been considered by Fogedby [6] via coupled Langevin equations

$$
\begin{aligned}
\frac{d X}{d s} & =\xi(s), \\
\frac{d t}{d s} & =\zeta(s),
\end{aligned}
$$

where $\xi(s)$ is a white Gaussian noise with $\langle\xi(s)\rangle=0$, $\left\langle\xi(s) \xi\left(s^{\prime}\right)\right\rangle=\delta\left(s-s^{\prime}\right)$, and $\zeta(s)$ is a white $\alpha$-stable Lévy noise, taking positive values only and independent of $\xi(s)$.

In (2), the random walk $x(t)$ is parametrized in terms of a continuous variable $s$, which is subjected to a random time change. This random time change to the physical time $t$ is described by the second equation. The combined process in the physical time is then given by $x(t)=X(s(t))$, where $s(t)$ is the inverse process to $t(s)$ defined as

$$
s(t)=\inf \{s: t(s)>t\} .
$$

Mathematically, the fundamental approach to describe the combined process $x(t)=X(s(t))$ is based on subordination technique, which was first introduced by Bochner [7]. Using the notation of subordination, the process $X(s), t(s)$, and $s(t)$ are named parent process, subordinator, and inverse subordinator, respectively.

In recent years, (2) consisting of Brownian motions with or without external field and inverse $\alpha$-stable subordinator are becoming a hot topic [8-17]. There are also several other processes considered as parent processes within the subordination framework, for example, Lévy-stable process 
$[18,19]$, arithmetic Brownian motion [20], geometric Brownian motion [21, 22], Ornstein-Uhlenbeck process [23, 24], tempered stable process [25], fractional Brownian motion $[26,27]$, and fractional Lévy-stable process [28]. Here, we note that, apart from inverse $\alpha$-stable subordinator, inverse tempered $\alpha$-stable subordinator and infinitely divisible subordinators are also considered in the literatures [16, 20, 2531].

In the simplest CTRW process, after each jumps, a new pair of waiting time and jump length is drawn from the associated distributions, independent of the previous values. This independence giving rise to a renewal process is not always justified, for instance, by observations of human motion patterns [32] and active biological movements [33] or in financial market dynamics [34]. Recently, three correlated CTRW models are introduced to model the random walks with some forms of memory [35-37]. Some advances in the field of CTRWs with correlated temporal or/and spatial structure can be also found in [38-45].

In this work, we consider a jump-correlated CTRW model which has the subordination form $x(t)=X\left(s_{\alpha}(t)\right)$. Here, the parent process $X(s)$ is an integrated Brownian motion, defined by

$$
X(s)=\int_{0}^{s} B\left(s^{\prime}\right) d s^{\prime}
$$

and inverse subordinator $s_{\alpha}(t)$ is the inverse of one-side $\alpha$ stable Lévy process $t(s)$, defined by

$$
s_{\alpha}(t)=\inf \{s>0: t(s)>t\} .
$$

The integrated Brownian motion $X(s)$ is called the random acceleration process in the physical literature and has been studied by many authors. For instance, it appears in the continuum description of the equilibrium Boltzmann weight of a semiflexible polymer chain [46]. It also appears in the description of statistical properties of the Burgers equation with Brownian initial velocity [47]. Some further results of the integrated Brownian motion can be found in the paper [48] reviewing this subject.

The structure of the paper is as follows. In Section 2, we introduce the jump-correlated CTRW model. In Section 3, we compute MSD of the proposed process and observe the corresponding anomalous diffusive behaviors. The timeaveraged MSD is also employed to show weak ergodicity breaking occurring in the proposed process. In Section 4, we generalize the integrated Brownian motion to the fractional integral of Brownian motion and compute the corresponding MSD. The conclusions are given in Section 5.

\section{Model}

We begin by recalling the general framework for CTRW theory. Let $\left\{T_{i}\right\}_{i \geq 1}$ be the sequence of nonnegative independent identically distributed (IID) random variable representing waiting times between jumps of a particle. We set $t(0)=0$ and $t(n)=\sum_{i=1}^{n} T_{i}$, that is, the time of the $n$th jump. Let $\left\{J_{i}\right\}_{i \geq 1}$ be the sequence of IID jump lengths of the particle, which are assumed to be independent of waiting times. We set $X(0)=0$ and $X(n)=\sum_{i=1}^{n} J_{i}$, that is, the position of the particle after the $n$th jump. Then, the position of the particle at time $t$ is given by

$$
x(t)=X(N(t))=\sum_{i=1}^{N(t)} J_{i}
$$

where $N(t)=\max \{n \geq 0: t(n) \leq t\}$ is the number of jumps up to time $t$. The process $x(t)=X(N(t))$ is called CTRW.

In what follows, we analyze a particular type of CTRW where the jump lengths are correlated. Assume that each jump is equal to

$$
J_{i}=\xi_{1}+\xi_{2}+\cdots+\xi_{i}
$$

where $\xi_{j}$ are IID random variables with finite second moment (for simplicity we assume that their second moment is equal to 1). Moreover, we assume that each waiting time $T_{i}$ is nonnegative IID random variable, whose characteristic function $\widehat{\varphi}(k)$ is given by

$$
\widehat{\varphi}(k)=\exp \left\{-|k|^{\alpha} \exp \left(-\frac{i \pi \alpha}{2} \operatorname{sgn}(k)\right)\right\},
$$

$$
0<\alpha \leq 1 \text {. }
$$

In the continuous limit, we get the following set of coupled Langevin equations for the position $x$ and time $t$ of the CTRW

$$
\begin{aligned}
\frac{d X(s)}{d s} & =\int_{0}^{s} \xi\left(s^{\prime}\right) d s^{\prime}=B(s), \\
\frac{d t(s)}{d s} & =\zeta(s),
\end{aligned}
$$

where $\xi(s)$ and $\zeta(s)$ are the same as those in $(2)$ and $B(s)$ is the standard Brownian motion with $\langle B(s)\rangle=0,\langle B(s) B(t)\rangle=$ $\min (s, t)$.

An equivalent representation of (9) in the form of subordination is

$$
x(t)=X\left(s_{\alpha}(t)\right) .
$$

Here the parent process $X(s)$ has the form

$$
X(s)=\int_{0}^{s} B\left(s^{\prime}\right) d s^{\prime},
$$

and the inverse subordinator $s_{\alpha}(t)$ is defined by

$$
s_{\alpha}(t)=\inf \{s>0: t(s)>t\},
$$

where $t(s)=\int_{0}^{s} \zeta\left(s^{\prime}\right) d s^{\prime}$ is an $\alpha$-stable totally skewed Lévy motion with characteristic function

$$
\left\langle e^{-u t(s)}\right\rangle=\exp \left\{-u^{\alpha} s\right\}, \quad 0<\alpha \leq 1 .
$$

\section{Discussions}

At first, let us compute the MSD of subordinated process $x(t)=X\left(s_{\alpha}(t)\right)$. 
Assume that $p(x, t), f(x, s)$, and $g(s, t)$ are PDFs of subordinated process $x(t)$, parent process $X(s)$, and inverse subordinator $s_{\alpha}(t)$, respectively. In terms of subordination, we have

$$
p(x, t)=\int_{0}^{\infty} f(x, s) g(s, t) d s .
$$

Since the first moment of parent process $X(s)$

$$
\langle X(s)\rangle=\left\langle\int_{0}^{s} B\left(s^{\prime}\right) d s^{\prime}\right\rangle=\int_{0}^{s}\left\langle B\left(s^{\prime}\right)\right\rangle d s^{\prime}=0
$$

and the second moment

$$
\begin{aligned}
\left\langle X^{2}(s)\right\rangle & =\left\langle\int_{0}^{s} B\left(s^{\prime}\right) d s^{\prime} \cdot \int_{0}^{s} B\left(s^{\prime \prime}\right) d s^{\prime \prime}\right\rangle \\
& =\int_{0}^{s} d s^{\prime} \int_{0}^{s}\left\langle B\left(s^{\prime}\right) B\left(s^{\prime \prime}\right)\right\rangle d s^{\prime \prime} \\
& =\int_{0}^{s} d s^{\prime} \int_{0}^{s} \min \left\{s^{\prime}, s^{\prime \prime}\right\} d s^{\prime \prime}=\frac{s^{3}}{3},
\end{aligned}
$$

we obtain

$$
\begin{aligned}
\langle x(t)\rangle & =\int_{0}^{\infty} x p(x, t) d x \\
& =\int_{0}^{\infty} d x \int_{0}^{\infty} x f(x, s) g(s, t) d s \\
& =\int_{0}^{\infty}\langle X(s)\rangle g(s, t) d s=0, \\
\left\langle x^{2}(t)\right\rangle & =\int_{-\infty}^{\infty} x^{2} p(x, t) d x \\
& =\int_{-\infty}^{\infty} d x \int_{0}^{\infty} x^{2} f(x, s) g(s, t) d s \\
& =\int_{0}^{\infty}\left\langle X^{2}(s)\right\rangle g(s, t) d s \\
& =\frac{1}{3} \int_{0}^{\infty} s^{3} g(s, t) d s .
\end{aligned}
$$

Thus, the MSD of the subordinated process $x(t)$ is

$$
\begin{aligned}
\left\langle(\Delta x)^{2}\right\rangle(t) & =\left\langle x^{2}(t)\right\rangle-\langle x(t)\rangle^{2} \\
& =\frac{1}{3} \int_{0}^{\infty} s^{3} g(s, t) d s .
\end{aligned}
$$

Let us turn to the inverse subordinator $s_{\alpha}(t)$. Observing the equivalence from (12)

$$
s_{\alpha} \leq s \Longleftrightarrow t(s)>t,
$$

we obtain the relation

$$
P\left(s_{\alpha} \leq s\right)=P(t(s)>t)=1-P(t(s) \leq t),
$$

which gives the formula for the PDF $g(s, t)$ in terms of the $\operatorname{PDF} h(t, s)$ :

$$
g(s, t)=-\frac{\partial}{\partial s} \int_{0}^{t} h\left(t^{\prime}, s\right) d t^{\prime} .
$$

Taking the Laplace transform for (22) about variable $t$, we get

$$
\widetilde{g}(s, u)=-\frac{\partial}{\partial s} \frac{1}{u} \widetilde{h}(u, s)=u^{\alpha-1} \exp \left\{-u^{\alpha} s\right\},
$$

$$
0<\alpha \leq 1 \text {. }
$$

Thus, the MSD of the subordinated process $x(t)$ in Laplace space is

$$
\begin{aligned}
\left\langle(\widetilde{\Delta x})^{2}\right\rangle(u) & =\frac{1}{3} \int_{0}^{\infty} s^{3} \widetilde{g}(s, u) d s \\
& =\frac{1}{3} \int_{0}^{\infty} s^{3} u^{\alpha-1} \exp \left\{-u^{\alpha} s\right\} d s=\frac{2}{u^{3 \alpha+1}},
\end{aligned}
$$

which implies that the MSD of $x(t)$ is

$$
\left\langle(\Delta x)^{2}\right\rangle(t)=\frac{2}{\Gamma(3 \alpha+1)} t^{3 \alpha}, \quad 0<\alpha \leq 1 .
$$

It is easy to observe from (25) that the process is subdiffusive when $0<\alpha<1 / 3$, normally diffusive when $\alpha=1 / 3$, and superdiffusive when $1 / 3<\alpha \leq 1$.

It is well-known that the MSD of the process given by (2) is of the form

$$
\left\langle(\Delta x)^{2}\right\rangle(t)=\frac{t^{\alpha}}{\Gamma(1+\alpha)} \quad 0<\alpha<1 .
$$

Comparing (25) with (26), we see that Fogedby's model can only represent anomalous subdiffusion, but our model can represent subdiffusion, normal diffusion, and superdiffusion.

Next, we study weak ergodicity breaking of the subordinated process $x(t)$.

In an ergodic system, one can find the equivalence

$$
\left\langle(\Delta x)^{2}\right\rangle(\Delta)=\left\langle\overline{\delta^{2}(\Delta)}\right\rangle .
$$

Here, $\overline{\delta^{2}(\Delta)}$ is the time-averaged MSD of the process $x(t)$, defined as

$$
\overline{\delta^{2}(\Delta)}=\frac{1}{T-\Delta} \int_{0}^{T-\Delta}[x(t+\Delta)-x(t)]^{2} d t,
$$

where $\Delta$ is the lag time and $T$ is the overall measure time.

For anomalous diffusion, the behavior of the ensemble $\operatorname{MSD}\left\langle(\Delta x)^{2}\right\rangle(\Delta)$ and the time-averaged MSD (28) may be fundamentally different. The disparity $\left\langle(\Delta x)^{2}\right\rangle(\Delta) \neq\left\langle\overline{\delta^{2}(\Delta)}\right\rangle$ is usually called weak ergodicity breaking (or weak nonergodicity) [49]. In recent years, weak nonergodicity of anomalous diffusion process attracts more and more attentions [49-55].

Since, for any $a>0$, parent process $X(s)$ satisfies

$$
X(a s)=\int_{0}^{a s} B\left(s^{\prime}\right) d s^{\prime}=a \int_{0}^{s} B(a \tau) d \tau={ }^{d} a^{3 / 2} X(s),
$$


where ${ }^{d}$ means an equality in distribution, we have

$$
\begin{aligned}
x(t) & =X\left(s_{\alpha}(t)\right)={ }^{d} X\left(t^{\alpha} s_{\alpha}(1)\right) \\
& ={ }^{d}\left(\frac{t^{3 \alpha}}{2}\right) X\left(s_{\alpha}(1)\right)=t^{3 \alpha / 2} x(1) .
\end{aligned}
$$

Thus,

$$
\begin{aligned}
& \left\langle\overline{\delta^{2}(\Delta)}\right\rangle=\frac{\left\langle x^{2}(1)\right\rangle}{T-\Delta} \int_{0}^{T-\Delta}\left[(t+\Delta)^{3 \alpha / 2}-t^{3 \alpha / 2}\right]^{2} d t \\
& =\frac{\left\langle x^{2}(1)\right\rangle}{T-\Delta} \int_{0}^{T-\Delta}\left[(t+\Delta)^{3 \alpha}+t^{3 \alpha}\right. \\
& \left.-2 t^{3 \alpha / 2}(t+\Delta)^{3 \alpha / 2}\right] d t \\
& =\frac{\left\langle x^{2}(1)\right\rangle}{T-\Delta}\left\{\frac { 1 } { ( 3 \alpha + 1 ) } \left[T^{3 \alpha+1}-\Delta^{3 \alpha+1}\right.\right. \\
& \left.\left.+(T-\Delta)^{3 \alpha+1}\right]-2 I_{1}\right\},
\end{aligned}
$$

where $I_{1}=\int_{0}^{T-\Delta} t^{3 \alpha / 2}(t+\Delta)^{3 \alpha / 2} d t$.

In the limit $\Delta \ll T$,

$$
\begin{aligned}
I_{1} & =\int_{0}^{T-\Delta} t^{3 \alpha / 2}(t+\Delta)^{3 \alpha / 2} d t \\
& =T^{3 \alpha+1} \int_{0}^{1-\Delta / T}\left(\tau+\frac{\Delta}{T}\right)^{3 \alpha / 2} \tau^{3 \alpha / 2} d \tau \\
& \simeq T^{3 \alpha+1} \int_{0}^{1-\Delta / T} \tau^{3 \alpha} d \tau=\frac{1}{3 \alpha+1}(T-\Delta)^{3 \alpha+1} .
\end{aligned}
$$

Hence,

$$
\begin{aligned}
& \left\langle\overline{\delta^{2}(\Delta)}\right\rangle \simeq \frac{\left\langle x^{2}(1)\right\rangle}{T-\Delta} \\
& \cdot \frac{1}{3 \alpha+1}\left[T^{3 \alpha+1}-\Delta^{3 \alpha+1}-(T-\Delta)^{3 \alpha+1}\right] \\
& =\frac{\left\langle x^{2}(1)\right\rangle}{T-\Delta} \frac{1}{3 \alpha+1} \\
& \cdot T^{3 \alpha+1}\left[1-\left(\frac{\Delta}{T}\right)^{3 \alpha+1}-\left(1-\frac{\Delta}{T}\right)^{3 \alpha+1}\right] \simeq\left\langle x^{2}(1)\right\rangle \\
& \cdot \frac{\Delta}{T^{1-3 \alpha}} .
\end{aligned}
$$

Since

$$
\left\langle(\Delta x)^{2}\right\rangle(\Delta)=\frac{2}{\Gamma(3 \alpha+1)} \Delta^{3 \alpha}, \quad 0<\alpha \leq 1,
$$

comparing (33) with (34), we see that the linear lag time dependence of $\left\langle\overline{\delta^{2}(\Delta)}\right\rangle$ is different from the power-law form $\Delta^{3 \alpha}$ of $\left\langle(\Delta x)^{2}\right\rangle(\Delta)$, which implies that subordinated process $x(t)$ is weakly nonergodic.
At last, we consider the propagator $p(x, t)$ associated with the subordinated process $x(t)$. By the total probability formula, we obtain an integral representation of $p(x, t)$ :

$$
p(x, t)=\int_{0}^{\infty} f(x, s) g(s, t) d s .
$$

For fixed $s>0$, the random variable $X(s)=\int_{0}^{s} B\left(s^{\prime}\right) d s^{\prime}$ is normally distributed. From (15) and (16), we have

$$
f(x, s)=\frac{\sqrt{3}}{\sqrt{2 \pi s^{3}}} \exp \left(-\frac{3 x^{2}}{2 s^{3}}\right) .
$$

It follows from

$$
\tilde{g}(s, u)=u^{\alpha-1} \exp \left\{-u^{\alpha} s\right\}, \quad 0<\alpha \leq 1,
$$

and the Laplace transform $s \mapsto q$ for $\widetilde{g}(s, u)$ that we obtain

$$
\tilde{\tilde{g}}(q, u)=\frac{u^{\alpha-1}}{u^{\alpha}+q} .
$$

After taking the inverse Laplace transform $u \mapsto t$ for $\tilde{\widetilde{g}}(q, u)$, we get

$$
\tilde{g}(q, t)=E_{\alpha}\left(-q t^{\alpha}\right),
$$

where

$$
E_{\alpha}(z)=\sum_{n=0}^{\infty} \frac{z^{n}}{\Gamma(n \alpha+1)}
$$

is the Mittag-Leffler function with parameter $\alpha$ [56].

\section{An Extension to the Fractional Case}

In this section, we introduce the dependent sequence of jump lengths $J_{i}$ in the following manner:

$$
J_{i}=\sum_{j=1}^{i} M(i-j+1) \xi_{j},
$$

where $M(\cdot)$ is a memory function. The continuous limit is of the form

$$
\begin{aligned}
\frac{d X(s)}{d s} & =\int_{0}^{s} M\left(s-s^{\prime}\right) \xi\left(s^{\prime}\right) d s^{\prime} \\
& =\int_{0}^{s} M\left(s-s^{\prime}\right) d B\left(s^{\prime}\right) .
\end{aligned}
$$

Integrating (42) we get

$$
X(s)=\int_{0}^{s} d s^{\prime} \int_{0}^{s^{\prime}} M\left(s^{\prime}-s^{\prime \prime}\right) d B\left(s^{\prime \prime}\right) .
$$

After taking $M(t)=t^{-\mu} / \Gamma(1-\mu)(0<\mu<1)$ and using the integration by parts, (43) can be written as

$$
X(s)=\frac{1}{\Gamma(1-\mu)} \int_{0}^{s} \frac{B\left(s^{\prime}\right)}{\left(s-s^{\prime}\right)^{\mu}} d s^{\prime}={ }_{0} I_{t}^{p} B(s),
$$


where ${ }_{0} I_{t}^{p}$ is the Riemann-Liouville fractional integration operator of order $p$, defined by [56]

$$
{ }_{0} I_{t}^{p} f(t)=\frac{1}{\Gamma(p)} \int_{0}^{t}(t-\tau)^{p-1} f(\tau) d \tau, \quad(p>0) .
$$

As a result, the jump-correlated CTRW has the subordination form $x(t)=X\left(s_{\alpha}(t)\right)$, where parent process $X(s)$ is of the form (44), and inverse subordinator $s_{\alpha}(t)$ is defined by (12).

Here, we are interested in the competition between the memory parameter $\mu$ and stability index $\alpha$. In what follows, we will not discuss any properties of motion other than the MSD.

In terms of (44), we get

$$
\begin{aligned}
& \left\langle X^{2}(s)\right\rangle=\frac{1}{\Gamma^{2}(1-\mu)} \\
& \cdot \int_{0}^{s} \frac{d s^{\prime}}{\left(s-s^{\prime}\right)^{\mu}} \int_{0}^{s} \frac{\left\langle B\left(s^{\prime}\right) B\left(s^{\prime \prime}\right)\right\rangle}{\left(s-s^{\prime \prime}\right)^{\mu}} d s^{\prime \prime} \\
& =\frac{1}{\Gamma^{2}(1-\mu)} \int_{0}^{s} \frac{d s^{\prime}}{\left(s-s^{\prime}\right)^{\mu}} \int_{0}^{s} \frac{\min \left\{s^{\prime}, s^{\prime \prime}\right\}}{\left(s-s^{\prime \prime}\right)^{\mu}} d s^{\prime \prime} \\
& =\frac{1}{\Gamma^{2}(1-\mu)} \int_{0}^{s} \frac{d s^{\prime}}{\left(s-s^{\prime}\right)^{\mu}}\left[\int_{0}^{s^{\prime}} \frac{s^{\prime \prime}}{\left(s-s^{\prime \prime}\right)^{\mu}} d s^{\prime \prime}\right. \\
& \left.+\int_{s^{\prime}}^{s} \frac{s^{\prime}}{\left(s-s^{\prime \prime}\right)^{\mu}} d s^{\prime \prime}\right] .
\end{aligned}
$$

By denoting $I(s)=\int_{0}^{s}\left(d s^{\prime} /\left(s-s^{\prime}\right)^{\mu}\right) \int_{0}^{s^{\prime}}\left(s^{\prime \prime} /\left(s-s^{\prime \prime}\right)^{\mu}\right) d s^{\prime \prime}$ and exchanging the order of quadratic integral $I(s)$, we obtain

$$
I(s)=\int_{0}^{s} \frac{d s^{\prime \prime}}{\left(s-s^{\prime \prime}\right)^{\mu}} \int_{s^{\prime \prime}}^{s} \frac{s^{\prime \prime}}{\left(s-s^{\prime}\right)^{\mu}} d s^{\prime} .
$$

Thus,

$$
\begin{aligned}
& \left\langle X^{2}(s)\right\rangle \\
& =\frac{2}{\Gamma^{2}(1-\mu)} \int_{0}^{s} \frac{s^{\prime}}{\left(s-s^{\prime}\right)^{\mu}} d s^{\prime} \int_{s^{\prime}}^{s} \frac{1}{\left(s-s^{\prime \prime}\right)^{\mu}} d s^{\prime \prime} \\
& =\frac{2}{(1-\mu) \Gamma^{2}(1-\mu)} \int_{0}^{s} s^{\prime}\left(s-s^{\prime}\right)^{1-2 \mu} d s^{\prime} \\
& =K_{\mu} s^{3-2 \mu}, \quad(0<\mu<1),
\end{aligned}
$$

where $K_{\mu}=2 B(2,2-2 \mu) /(1-\mu) \Gamma^{2}(1-\mu)$ and $B(a, b)=$ $\int_{0}^{1} x^{a-1}(1-x)^{b-1} d x$ is Beta function.

We observe from (48) that, in the limiting case $\mu \rightarrow 0$, memory function $M(t)=1$, the parent process $X(s)$ defined by (43) reduces to the form defined by (11), and the second moment of $X(s)$ computed by (48) reduces to $K_{0} s^{3}$, where $K_{0}=2 B(2,2)=1 / 3$, the same form as (16). We are also interested in the limiting case $\mu \rightarrow 1$. At the moment, the memory function $M(t)$ is a Dirac $\delta$-function; $X(s)$ defined by (43) reduces to the standard Brownian motion.

Let us turn to the MSD of the subordinated process $x(t)$. In terms of (18) and (48), we obtain

$$
\left\langle(\Delta x)^{2}\right\rangle(t)=\left\langle x^{2}(t)\right\rangle=K_{\mu} \int_{0}^{\infty} s^{3-2 \mu} g(s, t) d s
$$

In the Laplace space, the MSD is of the form

$$
\begin{aligned}
\left\langle(\widetilde{\Delta x})^{2}\right\rangle(u) & =K_{\mu} u^{\alpha-1} \int_{0}^{\infty} s^{3-2 \mu} e^{-u^{\alpha} s} d s \\
& =K_{\mu} \frac{\Gamma(4-2 \mu)}{u^{\alpha(3-2 \mu)+1}} .
\end{aligned}
$$

Taking the inverse Laplace transform for $\left\langle(\widetilde{\Delta x})^{2}\right\rangle(u)$, we have

$$
\left\langle(\Delta x)^{2}\right\rangle(t)=K_{\mu, \alpha} t^{\alpha(3-2 \mu)}, \quad 0<\mu<1,0<\alpha \leq 1,
$$

where $K_{\mu, \alpha}=K_{\mu} \Gamma(4-2 \mu) / \Gamma(\alpha(3-2 \mu)+1)$. In the limiting case $\mu \rightarrow 0$, the parameter $K_{\mu, \alpha}$ reduces to

$$
K_{0, \alpha}=\frac{K_{0} \Gamma(4)}{\Gamma(3 \alpha+1)}=\frac{2}{\Gamma(3 \alpha+1)} .
$$

Thus, (51) reduces to (25).

It is easy to observe from (51) that there exists a competition between the memory parameter $\mu$ and stability index $\alpha$. For the case $\alpha \leq 1 / 3$, the subordinated process exhibits subdiffusive behaviors. For the case $1 / 3<\alpha<1$, the process is subdiffusive when $1<3-2 \mu<1 / \alpha$, normal diffusive when $3-2 \mu=1 / \alpha$, and superdiffusive when $1 / \alpha<3-2 \mu<3$.

\section{Conclusions}

We introduce an integrated Brownian motion subordinated by inverse $\alpha$-stable one-sided Lévy motion, which is a continuous limit of a jump-correlated CTRW. In terms of the ensemble MSD of the proposed process, we conclude that the process is subdiffusive when $0<\alpha<1 / 3$, normal diffusive when $\alpha=1 / 3$, and superdiffusive when $1 / 3<\alpha \leq 1$. The time-averaged MSD is also employed to show weak ergodicity breaking occurring in the proposed process.

We also generalize the process to the case, where the parent process is fractional integral of Brownian motion. In terms of the MSD, we observe a competition between the memory parameter $\mu$ and stability index $\alpha$. Other types of inverse subordinators may be also candidates.

\section{Conflicts of Interest}

The authors declare that there are no conflicts of interest regarding the publication of this paper.

\section{Acknowledgments}

This work is supported by the National Natural Science Foundation of China (Grant no. 11671343) and the Scientific Research Project of Hunan Provincial Education Department (no. 17B258). 


\section{References}

[1] J.-P. Bouchaud and A. Georges, "Anomalous diffusion in disordered media: statistical mechanisms, models and physical applications," Physics Reports, vol. 195, no. 4-5, pp. 127-293, 1990.

[2] R. Metzler and J. Klafter, "The random walk's guide to anomalous diffusion: a fractional dynamics approach," Physics Reports, vol. 339, no. 1, pp. 1-77, 2000.

[3] R. Metzler and J. Klafter, "The restaurant at the end of the random walk: recent developments in the description of anomalous transport by fractional dynamics," Journal of Physics. A. Mathematical and General, vol. 37, no. 31, pp. R161-R208, 2004.

[4] I. Eliazar and J. Klafter, "Anomalous is ubiquitous," Annals of Physics, vol. 326, no. 9, pp. 2517-2531, 2011.

[5] E. W. Montroll and G. H. Weiss, "Random walks on lattices. II," Journal of Mathematical Physics, vol. 6, pp. 167-181, 1965.

[6] H. C. Fogedby, "Langevin equations for continuous time Lévy flights," Physical Review E, vol. 50, no. 2, pp. 1657-1660, 1994.

[7] S. Bochner, "Diffusion equation and stochastic processes," Proceedings of the National Academy of Sciences, vol. 35, no. 7, pp. 368-370, 1949.

[8] A. Baule and R. Friedrich, "Joint probability distributions for a class of non-Markovian processes," Physical Review E, vol. 71, no. 2, Article ID 026101, 2005.

[9] A. Piryatinska, A. I. Saichev, and W. A. Woyczynski, "Models of anomalous diffusion: the subdiffusive case," Physica A: Statistical Mechanics and Its Applications, vol. 349, no. 3-4, pp. 375-420, 2005.

[10] M. Magdziarz, A. Weron, and K. Weron, "Fractional FokkerPlanck dynamics: stochastic representation and computer simulation," Physical Review E-Statistical, Nonlinear, and Soft Matter Physics, vol. 75, no. 1, Article ID 016708, 2007.

[11] D. Kleinhans and R. Friedrich, "Continuous-time random walks: simulation of continuous trajectories," Physical Review E, vol. 76, no. 6, Article ID 061102, 2007.

[12] A. Weron, M. Magdziarz, and K. Weron, "Modeling of subdiffusion in space-time-dependent force fields beyond the fractional Fokker-Planck equation," Physical Review E, vol. 77, no. 3, Article ID 036704, 2008.

[13] M. Magdziarz, A. Weron, and J. Klafter, "Equivalence of the fractional fokker-planck and subordinated langevin equations: the case of a time-dependent force," Physical Review Letters, vol. 101, no. 21, Article ID 210601, 2008.

[14] A. Weron and S. Orzel, "Itô formula for subordinated Langevin equation. A case of time dependent force," Acta Physica Polonica $B$, vol. 40, no. 5, pp. 1271-1277, 2009.

[15] S. Eule and R. Friedrich, "Subordinated Langevin equations for anomalous diffusion in external potentials-biasing and decoupled external forces," EPL, vol. 86, no. 3, Article ID 30008, 2009.

[16] M. Magdziarz, "Langevin picture of subdiffusion with infinitely divisible waiting times," Journal of Statistical Physics, vol. 135, no. 4, pp. 763-772, 2009.

[17] M. Magdziarz, "Stochastic representation of subdiffusion processes with time-dependent drift," Stochastic Processes and Their Applications, vol. 119, no. 10, pp. 3238-3252, 2009.

[18] M. Magdziarz and A. Weron, "Competition between subdiffusion and Lévy flights: a Monte Carlo approach," Physical Review E, vol. 75, Article ID 056702, 2007.

[19] B. o. Dybiec and E. Gudowska-Nowak, "Subordinated diffusion and continuous time random walk asymptotics," Chaos, vol. 20, no. 4, Article ID 043129, 2010.
[20] A. Wyłomańska, "Arithmetic Brownian motion subordinated by tempered stable and inverse tempered stable processes," Physica A: Statistical Mechanics and Its Applications, vol. 391, no. 22, pp. 5685-5696, 2012.

[21] H. Gu, J.-R. Liang, and Y.-X. Zhang, "On a time-changed geometric Brownian motion and its application in financial market," Acta Physica Polonica B, vol. 43, no. 8, pp. 1667-1681, 2012.

[22] J. Gajda and A. Wyłomańska, "Geometric Brownian motion with tempered stable waiting times," Journal of Statistical Physics, vol. 148, no. 2, pp. 296-305, 2012.

[23] J. Janczura, S. Orzeł, and A. Wyłomańska, "Subordinated $\alpha \alpha$ stable Ornstein-Uhlenbeck process as a tool for financial data description," Physica A: Statistical Mechanics and its Applications, vol. 390, no. 23-24, pp. 4379-4387, 2011.

[24] J. Gajda and A. Wyłomańska, "Time-changed Ornstein-Uhlenbeck process," Journal of Physics A: Mathematical and Theoretical, vol. 48, no. 13, Article ID 135004, 2015.

[25] A. Wyłomańska, "The tempered stable process with infinitely divisible inverse subordinators," Journal of Statistical Mechanics: Theory and Experiment, vol. 2013, no. 10, Article ID P10011, 2013.

[26] Y.-X. Zhang, H. Gu, and J.-R. Liang, "Fokker-planck type equations associated with subordinated processes controlled by tempered $\alpha$-stable processes," Journal of Statistical Physics, vol. 152, no. 4, pp. 742-752, 2013.

[27] J. Gajda and A. Wyłomańska, "Fokker-Planck type equations associated with fractional Brownian motion controlled by infinitely divisible processes," Physica A: Statistical Mechanics and Its Applications, vol. 405, pp. 104-113, 2014.

[28] M. Teuerle, A. Wyłomańska, and G. Sikora, "Modeling anomalous diffusion by a subordinated fractional Lévy-stable process," Journal of Statistical Mechanics: Theory and Experiment, vol. 2013, no. 5, Article ID P05016, 2013.

[29] J. Gajda and M. Magdziarz, "Fractional Fokker-Planck equation with tempered $\alpha$-stable waiting times: Langevin picture and computer simulation," Physical Review E. Statistical, Nonlinear, and Soft Matter Physics, vol. 82, no. 1, Article ID 011117, 2010.

[30] J. Janczura and A. Wyłomańska, "Anomalous diffusion models: different types of subordinator distribution," Acta Physica Polonica B, vol. 43, no. 5, pp. 1001-1016, 2012.

[31] J. Gajda, "Fractional Fokker-Planck equation with space dependent drift and diffusion: the case of tempered $\alpha$-stable waitingtimes," Jagellonian University. Institute of Physics. Acta Physica Polonica B, vol. 44, no. 5, pp. 1149-1161, 2013.

[32] C. Song, T. Koren, P. Wang, and A. Barabási, "Modelling the scaling properties of human mobility," Nature Physics, vol. 6, no. 10, pp. 818-823, 2010.

[33] A. Maye, C.-H. Hsieh, G. Sugihara, and B. Brembs, "Order in spontaneous behavior," PLoS ONE, vol. 2, no. 5, article e443, 2007.

[34] E. Scalas, "The application of continuous-time random walks in finance and economics," Physica A: Statistical Mechanics and its Applications, vol. 362, no. 2, pp. 225-239, 2006.

[35] A. Chechkin, M. Hofmann, and I. M. Sokolov, "Continuoustime random walk with correlated waiting times," Physical Review E. Statistical, Nonlinear, and Soft Matter Physics, vol. 80, no. 3, Article ID 031112, 2009.

[36] M. M. Meerschaert, E. Nane, and Y. Xiao, "Correlated continuous time random walks," Statistics \& Probability Letters, vol. 79, no. 9, pp. 1194-1202, 2009. 
[37] V. Tejedor and R. Metzler, "Anomalous diffusion in correlated continuous time random walks," Journal of Physics. A. Mathematical and Theoretical, vol. 43, no. 8, Article ID 082002, 2010.

[38] M. Magdziarz, R. Metzler, W. Szczotka, and P. Zebrowski, "Correlated continuous-time random walks in external force fields," Physical Review E, vol. 85, no. 5, Article ID 051103, 2012.

[39] M. Magdziarz, R. Metzler, W. Szczotka, and P. Zebrowski, "Correlated continuous-time random walks-scaling limits and Langevin picture," Journal of Statistical Mechanics: Theory and Experiment, vol. 2012, no. 4, Article ID P04010, 2012.

[40] M. Magdziarz, W. Szczotka, and P. Zebrowski, "Asymptotic behaviour of random walks with correlated temporal structure," Proceedings of the Royal Society A: Mathematical, Physical and Engineering Sciences, vol. 469, no. 2159, Article ID 20130419, 2013.

[41] J. H. Schulz, A. V. Chechkin, and R. Metzler, "Correlated continuous time random walks: combining scale-invariance with long-range memory for spatial and temporal dynamics," Journal of Physics. A. Mathematical and Theoretical, vol. 46, no. 47, Article ID 475001, 2013.

[42] L. Shi, Z. Yu, H. Huang, Z. Mao, and A. Xiao, "The subordinated processes controlled by a family of subordinators and corresponding Fokker-Planck type equations," Journal of Statistical Mechanics: Theory and Experiment, vol. 2014, no. 12, Article ID P12002, 2014.

[43] J. Wang, J. Zhou, L. Lv, W. Qiu, and F. Ren, "Heterogeneous memorized continuous time random walks in an external force fields," Journal of Statistical Physics, vol. 156, no. 6, pp. 1111-1124, 2014.

[44] F.-Y. Ren, J. Wang, L.-J. Lv, H. Pan, and W.-Y. Qiu, "Effect of different waiting time processes with memory to anomalous diffusion dynamics in an external force fields," Physica A: Statistical Mechanics and Its Applications, vol. 417, pp. 202-214, 2015.

[45] L. Lv, F.-Y. Ren, J. Wang, and J. Xiao, "Correlated continuous time random walk with time averaged waiting time," Physica A. Statistical Mechanics and Its Applications, vol. 422, pp. 101-106, 2015.

[46] T. W. Burkhardt, "Semiflexible polymer in the half plane and statistics of the integral of a Brownian curve," Journal of Physics A: Mathematical and General, vol. 26, no. 22, pp. L1157-L1162, 1993.

[47] P. Valageas, "Statistical properties of the burgers equation with brownian initial velocity," Journal of Statistical Physics, vol. 134, no. 3, pp. 589-640, 2009.

[48] T. W. Burkhardt, "The random acceleration process in bounded geometries," Journal of Statistical Mechanics: Theory and Experiment, vol. 2007, no. 7, Article ID P07004, 2007.

[49] J. Jeon, A. V. Chechkin, and R. Metzler, "Scaled Brownian motion: a paradoxical process with a time dependent diffusivity for the description of anomalous diffusion," Physical Chemistry Chemical Physics, vol. 16, no. 30, pp. 15811-15817, 2014.

[50] Y. He, S. Burov, R. Metzler, and E. Barkai, "Random time-scale invariant diffusion and transport coefficients," Physical Review Letters, vol. 101, no. 5, Article ID 058101, 2008.

[51] W. Deng and E. Barkai, "Ergodic properties of fractional Brownian-Langevin motion," Physical Review E, vol. 79, no. 1, Article ID 011112, 2009.

[52] J.-H. Jeon and R. Metzler, "Fractional Brownian motion and motion governed by the fractional Langevin equation in confined geometries," Physical Review E, vol. 81, no. 2, Article ID 021103, 2010.
[53] E. Barkai, Y. Garini, and R. Metzler, "Strange kinetics of single molecules in living cells," Physics Today, vol. 65, no. 8, pp. 29-35, 2012.

[54] J. Kursawe, J. Schulz, and R. Metzler, "Transient aging in fractional Brownian and Langevin-equation motion," Physical Review E, vol. 88, no. 6, Article ID 062124, 2013.

[55] R. Metzler, J.-H. Jeon, A. G. Cherstvy, and E. Barkai, "Anomalous diffusion models and their properties: non-stationarity, non-ergodicity, and ageing at the centenary of single particle tracking," Physical Chemistry Chemical Physics, vol. 16, no. 44, pp. 24128-24164, 2014.

[56] I. Podlubny, Fractional Differential Equations, Academic Press, San Diego, Calif, USA, 1999. 


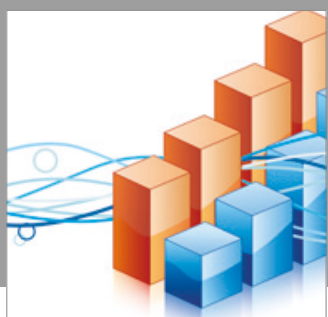

Advances in

Operations Research

vatersals

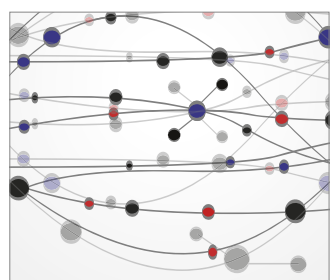

\section{The Scientific} World Journal
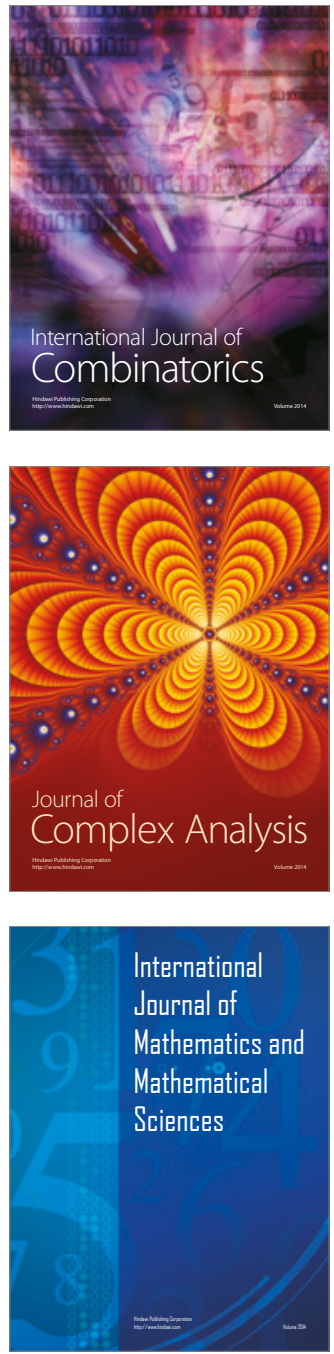
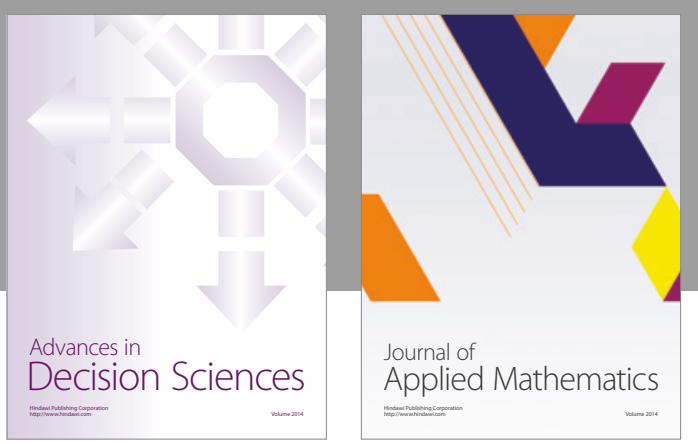

Algebra

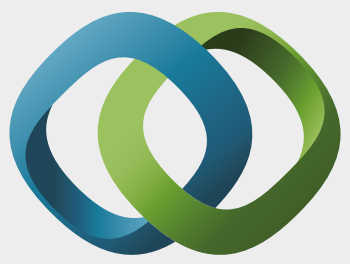

\section{Hindawi}

Submit your manuscripts at

https://www.hindawi.com
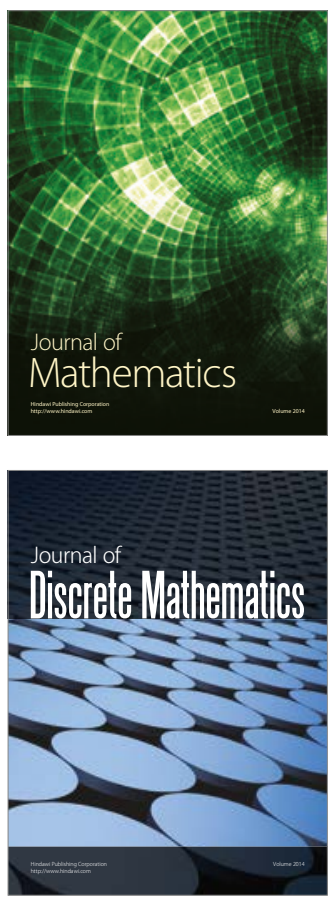

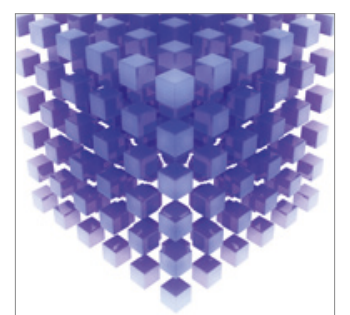

Mathematical Problems in Engineering
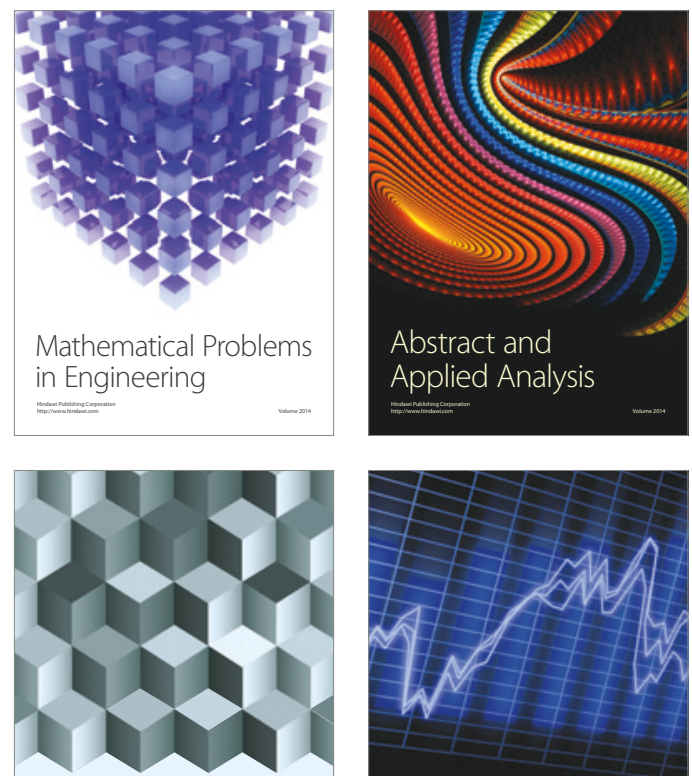

Journal of

Function Spaces

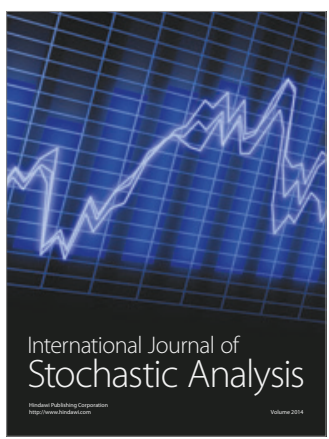

Probability and Statistics
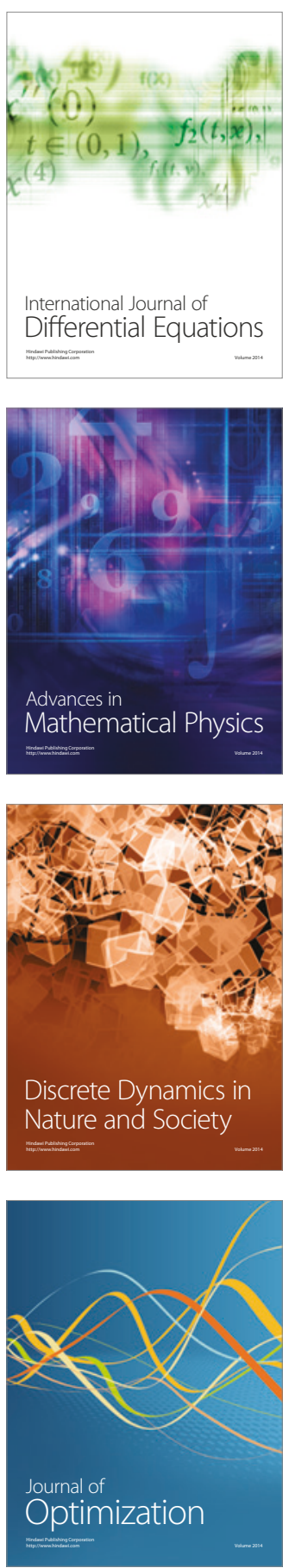